\title{
The SCIAMACHY cloud products: Algorithms and examples from ENVISAT
}

\author{
A.A. Kokhanovsky *, V.V. Rozanov, J.P. Burrows, K.-U. Eichmann, \\ W. Lotz, M. Vountas \\ Institute of Environmental Physics, University of Bremen, D-28334 Bremen, Germany
}

Received 29 September 2004; received in revised form 2 March 2005; accepted 3 March 2005

\begin{abstract}
We present here a newly developed satellite cloud retrieval algorithm SACURA. The algorithm is based on the modified exponential approximation for the cloud reflection function. It is valid for clouds having the optical thickness larger than 5. Retrieval results for selected cloudy fields using data from SCIAMACHY onboard ENVISAT are presented. The cloud algorithm itself does not use the look-up-table approach and can be applied to other instruments placed on satellite and airborne platforms.
\end{abstract}

(C) 2005 COSPAR. Published by Elsevier Ltd. All rights reserved.

Keywords: Clouds; Terrestrial atmosphere; Radiative transfer; Remote sensing

\section{Introduction}

Clouds play an important role in the Earth climate system (Kondratyev and Binenko, 1984; Liou, 1992). The amount of radiation reflected by the Earth-atmosphere system into outer space depends not only on the cloud cover and the total amount of condensed water in the Earth atmosphere but also on the size of droplets $a_{\text {ef }}$, the thermodynamic state of water in clouds, and vertical distributions of cloud parameters. The information about microphysical properties and spatial distributions of terrestrial clouds on a global scale can be obtained only with satellite remote sensing systems. Different spectrometers and radiometers (Bovensmann et al., 1999, 2004; Deschamps et al., 1994; King et al., 1992; Nakajima et al., 1998), deployed on space-based platforms, measure the angular and spectral distribution

\footnotetext{
* Corresponding author. Tel.: +49 421244 5628; fax: +49 4212184555.

E-mail address: alexk@iup.physik.uni-bremen.de (A.A. Kokhanovsky).
}

of intensity and polarization of backscattered solar light. Generally, the measured values depend on both geometrical and microphysical characteristics of clouds. Thus, the inherent properties of clouds can be retrieved (at least in principle) solving the inverse problem. The accuracy of the retrieved values depends on the accuracy of measurements and forward radiative transfer model. In particular, it is often assumed that clouds can be represented as homogeneous and infinitely extended in the horizontal direction plane-parallel slabs (Goloub et al., 2000; Han et al., 1994; King, 1987; Nakajima and King, 1990; Nakajima et al., 1991; Rossow, 1989; Rossow and Schiffer, 1999; Kokhanovsky et al., 2003). The range of applicability of such an assumption for real clouds is very limited as is shown by observations of light from the sky on a cloudy day. For example, it was found that the retrieved cloud optical thickness $\tau$ depends on the viewing geometry (Loeb and Davies, 1996; Loeb and Coakley, 1998). This, of course, would not be the case for an idealized plane-parallel cloud layer. However, both the state-of-art radiative transfer theory and computer technology are not capable to incorporate three 
dimensional effects into operational satellite retrieval schemes. As a result, cloud parameters retrieved should be considered as a rather coarse approximation to reality. However, even such limited tools produce valuable information on terrestrial clouds properties. For example, it was confirmed by satellite measurements that droplets in clouds over oceans are usually larger than those over land (Han et al., 1994). This feature, for instance, is of importance for the simulation of the Earth's climate (Slingo, 1989). Microphysical characteristics of clouds are of importance for dynamical meteorology. For example, the probability of precipitation increases for larger effective radii.

A new Semi-Analytical CloUd Retrieval Algorithm (SACURA) for the cloud liquid water path (LWP) and water droplets size determination presented here is based on the asymptotical solution of the radiative transfer equation for a special case of disperse media, having large optical thickness. This solution was obtained by Germogenova $(1961,1963)$ for plane-parallel turbid slabs. Such an approach has already been used in a number of studies (Rozenberg et al., 1978; King, 1987). The difference is that the asymptotical solutions are further simplified such that the inverse problem is reduced to the solution of a single transcendent equation. This allows us to speed up the retrieval process significantly without the substantial loss of the accuracy of the retrieved cloud characteristics.

The algorithm is restricted to the case of optically thick clouds (the optical thickness $\tau \geqslant 5$ ). It is planned to be supplemented in future by the look-up-table (LUT) approach at $\tau<5$ (Nakajima and King, 1990). However, it should be stressed that the optical thickness is highly correlated with the geometrical thickness of clouds. For instance, it was shown (Feigelson, 1981) that clouds having $\tau<5$ have the geometrical thickness less than $200 \mathrm{~m}$ on average. It is difficult to expect that such clouds are homogeneous in the horizontal direction. Thus, the horizontal photon transport must be accounted for (Platnick, 2001). However, this is not considered in standard retrieval procedures (Arking and Childs, 1985; Nakajima and King, 1990; Platnick et al., 2001, 2003; Nakajima et al., 2005). So results obtained from more accurate forward modelling as compared to ours are anyway biased due to cloud inhomogeneity effects. This gives another justification besides speed concerns to the approach we use.

Besides $a_{\text {ef }}$ and $\tau$, we also retrieve the liquid water path $w\left(\mathrm{gm}^{-2}\right)$, the cloud albedo $r$, and the column concentration of droplets $N\left(\mathrm{~m}^{-2}\right)$, using well-known relationships between these quantities (see below).

The cloud top height is derived from measurements in the oxygen A-absorption band as recommended by Yamomoto and Wark (1961). Such an approach has been used extensively by many authors (Kuze and
Chance, 1994; Fischer et al., 2000; Koelemeijer et al., 2001, 2002; Kuji and Nakajima, 2002).

Finally, the cloud thermodynamic state is obtained using the fact that spectral signatures of liquid water differ from those for ice in the selected spectral range 1550 $1670 \mathrm{~nm}$ (Pilewskie and Twomey, 1987a,b; Knap et al., 2002; Acarreta et al., 2004).

\section{The determination of the cloud optical thickness, the cloud albedo, the liquid water path, the column droplet concentration, and the effective size of particles using backscattered solar light measurements}

The SACURA is based on the two-wavelength semianalytical cloud retrieval algorithm for the liquid water path $w$ and the cloud particle size $a_{\text {ef }}$ determination proposed by Kokhanovsky et al. (2003). It is known that the reflection function in visible is primarily dependent on the cloud optical thickness (and, therefore, on the LWP) and the infrared channels are sensitive to the size of particles in clouds (Nakajima and King, 1990).

Parameters $a_{\mathrm{ef}}$ and $w$ are used to find the cloud optical thickness $\tau$, the cloud albedo $r$, and the column droplet concentration $N\left(\mathrm{~cm}^{-2}\right)$ from following approximate analytical equations valid in the visible (Kokhanovsky, 2004a,b):

$$
\begin{aligned}
& \tau=\frac{1.5 w}{\rho a_{\mathrm{ef}}}\left\{1+\frac{1.1}{\left(k a_{\mathrm{ef}}\right)^{2 / 3}}\right\}, \quad r=\frac{1}{1.07+0.75 \tau(1-g)}, \\
& N=\frac{\tau}{2 \pi \zeta a_{\mathrm{ef}}^{2}},
\end{aligned}
$$

where $\rho=1 \mathrm{~g} \mathrm{~cm}^{-3}$ is the density of water, $k=2 \pi / \lambda, \lambda$ is the wavelength, $\zeta=0.7$ and

$g=0.08-0.5\left(k a_{\mathrm{ef}}\right)^{-2 / 3}$,

is the asymmetry parameter.

The values of $a_{\mathrm{ef}}$ and $w$ are found from the system of two algebraic equations (Kokhanovsky et al., 2003; Kokhanovsky, 2004c):

$$
\begin{aligned}
R_{1}= & R_{\infty}^{0}-\frac{t_{1}\left(a_{\mathrm{ef}}, w\right)\left[1-A_{1}\right]}{1-A_{1}\left[1-t_{1}\left(a_{\mathrm{ef}}, w\right)\right]} K_{0}(\vartheta) K_{0}\left(\vartheta_{0}\right), \\
R_{2}= & R_{\infty}^{0} \exp \left(-y_{2}\left(a_{\mathrm{ef}}\right)\left(1-0.05 y_{2}\left(a_{\mathrm{ef}}\right)\right) u\right) \\
& -\left[\exp \left(-x_{2}\left(a_{\mathrm{ef}}, w\right)-y_{2}\left(a_{\mathrm{ef}}\right)\right)-\frac{t_{2}\left(a_{\mathrm{ef}}, w\right) A_{2}}{1-A_{2} r_{2}\left(a_{\mathrm{ef}}, w\right)}\right] \\
& \times t_{2}\left(a_{\mathrm{ef}}, w\right) K_{0}(\vartheta) K_{0}\left(\vartheta_{0}\right) .
\end{aligned}
$$

The subscripts " 1 " and " 2 " refer to wavelengths $\lambda_{1}$ and $\lambda_{2}$ in visible and near - infrared channels, respectively. $R_{1}$ and $R_{2}$ are measured reflection functions in the visible and infrared, respectively. Specifically, the SACURA uses measurements at the wavelengths 443 and $1550 \mathrm{~nm}$. These channels are almost free of gaseous absorption. 
Table 1

Auxiliary functions (Kokhanovsky and Rozanov, 2003; Kokhanovsky et al., 2003; Kokhanovsky, 2004a; Van de Hulst, 1980)

\begin{tabular}{ll}
\hline The auxiliary function & Formula \\
\hline$u$ & $\frac{K_{0}(\vartheta) K_{0}\left(\vartheta_{0}\right)}{R_{\infty}^{0}\left(\vartheta, \vartheta_{0}, \varphi\right)}$ \\
$K_{0}(\vartheta)$ & $\frac{3}{7}(1+2 \cos \vartheta)$ \\
$R_{\infty}^{0}$ & $\frac{3.944-2.5\left(\cos \vartheta_{0}+\cos \vartheta\right)+10.664 \cos \vartheta_{0} \cos \vartheta+F(\theta)}{4\left(\cos \vartheta+\cos \vartheta_{0}\right)}$ \\
$t_{2}$ & $\frac{\sinh y_{2}}{\sinh \left[x_{2}+1.07 y_{2}\right]}-\frac{4.86-13.08 \cos \vartheta_{0} \cos \vartheta+12.76 \cos \vartheta_{0}^{2} \cos \vartheta^{2}}{\tau^{3}} \exp \left[x_{2}\right]$ \\
$r_{2}$ & $\exp \left(-y_{2}\left(1-0.05 y_{2}\right)\right)-t_{2} \exp \left(-x_{2}-y_{2}\right)$ \\
$x_{2}$ & $\gamma_{2} \tau$ \\
$y_{2}$ & $4 \gamma_{2} / 3\left(1-g_{2}\right)$ \\
$\gamma_{2}$ & $\sqrt{3\left(1-g_{2}\right)\left(1-\omega_{o 2}\right)}$ \\
\hline
\end{tabular}

Here, $R_{\infty}^{0}$ is the reflection function of a semiinfinite nonabsorbing cloud layer, $K_{0}(\vartheta)$ is the escape function, which describes the angular distribution of light transferred by a thick cloud layer, $t_{j}$ and $r_{j}$ are hemispherical cloud transmittance and reflectance, respectively, for diffuse light illumination conditions, and $\gamma_{j}$ is the diffusion exponent. The index $j=1,2$ corresponds to the spectral channel used. The function $F(\theta)$ is calculated as $F(\theta)=\sum_{m=1}^{\infty} x_{m}\left[P_{m}(\cos \theta)-(-1)^{m} P_{m}(\cos \vartheta) P_{m}\left(\cos \vartheta_{0}\right)\right]$, where $x_{m}$ are coefficients of the expansion of the phase function with respect to Legendre polynomials $P_{m}(\cos \theta)$ (Kokhanovsky, 2004b). Here $\theta=\cos ^{-1}\left(-\cos \vartheta \cos \vartheta_{0}+\sin \vartheta \sin \vartheta_{0} \cos \varphi\right)$ is the scattering angle, $\vartheta_{0}$ is the solar zenith angle, $\vartheta$ is the observation zenith angle, and $\varphi$ is the relative azimuth. The value of $t_{1}$ can be calculated from $r$ given by Eq. (1) at the wavelength $\lambda_{2}$. Namely, it follows: $t_{1}=1-r\left(\lambda_{1}\right)$.

Values of $A_{1}$ and $A_{2}$ give us the surface albedos in the visible and near-infrared, respectively, $\vartheta_{0}$ is the solar zenith angle, $\vartheta$ is the zenith observation angle. Other functions in Eqs. (3) and (4) are given in Table 1.

We underline that the land surface reflectance generally decreases for shorter wavelengths. This allows to neglect its influence on retrievals for optically thick clouds considered in this paper at the wavelength $443 \mathrm{~nm}$. Our simulations show that for bright surfaces like snow and ice the surface reflectance must be accounted for in the retrieval procedures even for relatively optically thick clouds having values of $\tau$ below 70 for water clouds and 40 for ice clouds.

Eqs. (3) and (4) have two unknowns ( $a_{\text {ef }}$ and $w$ ). Standard methods and programs are available for the solution of such problems. However, taking into account the specific form of Eq. (3), the exclusion method is used here. So we can formulate a single transcendent equation with one unknown $\left(a_{\mathrm{ef}}\right)$. It follows from Eq. (3) that

$t_{1}=\left\{\frac{K_{0}\left(\vartheta_{0}\right) K_{0}(\vartheta)}{R_{\infty}^{0}-R_{1}}-\frac{A_{1}}{1-A_{1}}\right\}^{-1}$.

On the other hand, it follows from the formula for $t_{1}$ given in Table 1 in the visible:

$\tau_{1}=\frac{4\left(t_{1}^{-1}-1.07\right)}{3\left(1-g\left(a_{\mathrm{ef}}\right)\right)}$

where we neglect $\Delta$ (see Table 1 ) for the sake of simplicity and the parameter $g_{1}$ is given by Eq. (2) at $\lambda=\lambda_{1}$.

The optical thickness $\tau_{1}$ is related to the liquid water path $w$ and the effective radius of droplets by the following equation (see Eq. (1)):

$\tau_{1}=w \bar{\sigma}_{\text {ext }}\left(\lambda_{1}, a_{\mathrm{ef}}\right)$, where

$\bar{\sigma}_{\mathrm{ext}}=\frac{1.5}{\rho a_{\mathrm{ef}}}\left(1+\frac{1.1}{\left(k a_{\mathrm{ef}}\right)^{2 / 3}}\right)$.

Therefore, the analytical expression for $w$ can be obtained from Eqs. (6)-(8):

$w=\frac{4\left(t_{1}^{-1}-1.07\right)}{3\left(1-g_{1}\left(a_{\mathrm{ef}}\right)\right) \bar{\sigma}_{\mathrm{ext}}\left(\lambda_{1}, a_{\mathrm{ef}}\right)}$,

where the dependence of all functions on the value of the effective radius is explicitly noted. Thus, one unknown parameter $(w)$ is expressed in terms of another $\left(a_{\mathrm{ef}}\right)$. The substitution of Eq. (9) into Eq. (4) with account for formulae in Table 1 yields a single transcendent equation for the determination of the effective radius of droplets in a cloud. It should be pointed out that $w$ enters Eq. (4) via $x_{2}\left(a_{\mathrm{ef}}, w\right)$. The parameter $y_{2}=4 \sqrt{\frac{1-\omega_{02}}{3\left(1-g_{2}\right)}}$ in Eq. (4) does not depend on $w$ by definition (see Table 1). Here, $\omega_{02}$ and $g_{2}$ are the single scattering albedo and the asymmetry parameter at $\lambda=\lambda_{2}$, respectively. The function $x_{2}\left(a_{\mathrm{ef}}, w\right)$ can be written as :

$x_{2}\left(a_{\mathrm{ef}}, w\right)=\sqrt{3\left(1-\omega_{02}\left(a_{\mathrm{ef}}\right)\right)\left(1-g_{2}\left(a_{\mathrm{ef}}\right)\right)} \tau_{2}\left(a_{\mathrm{ef}}, w\right)$,

where only $\tau_{2}$ depends on the liquid water path $w$. The index " 2 " corresponds to the wavelength $\lambda_{2}$ in the near-infrared. We use following results valid at $\lambda_{2}=1550 \mathrm{~nm}$ :

$1-g_{2}=\sum_{i=0}^{4} c_{i}\left(2 \pi a_{\mathrm{ef}} / \lambda_{2}\right)^{-2 i / 3}$,

$\omega_{0}=\frac{4 \pi \chi}{\lambda_{2} \rho \bar{\sigma}_{\mathrm{ext}}\left(\lambda_{2}, a_{\mathrm{ef}}\right)} \sum_{i=0}^{4} d_{i}\left(2 \pi a_{\mathrm{ef}} / \lambda_{2}\right)^{i}$, 
Table 2

Parameters $c_{i}$ and $d_{i}$ in Eq. (11)

\begin{tabular}{llllll}
\hline$i$ & 0 & 1 & 2 & 3 & \multicolumn{1}{l}{4} \\
\hline$c_{i}$ & 0.0608 & 2.465 & -32.98 & 248.94 & -636 \\
$d_{i}$ & 1.671 & 0.0025 & -0.0002365 & $2.86 \mathrm{e}-6$ & $-1 . \mathrm{e}-8$ \\
\hline
\end{tabular}

where parameters $c_{i}$ and $d_{i}$ are given in Table 2 . These expressions were obtained fitting Mie results for spherical polydispersions with account for general geometrical optics results for large spherical droplets (Kokhanovsky, 2004a).

Taking into account the definition of the optical thickness: $\tau=\sigma_{\text {ext }} L$, where $L$ is the cloud geometrical thickness and $\sigma_{\text {ext }}$ is the extinction coefficient, $\tau_{2}$ in Eq. (10) is given by: $\tau_{2}=\Phi\left(a_{\mathrm{ef}}\right) \tau_{1}$. The analytical expression for the function

$\Phi\left(a_{\mathrm{ef}}\right)=\frac{\bar{\sigma}_{\mathrm{ext}}\left(\lambda_{2}\right)}{\bar{\sigma}_{\mathrm{ext}}\left(\lambda_{1}\right)}$,

is easily derived from Eq. (8).

Summing up, the substitution of Eq. (10) and also other parameters from Table 1 into Eq. (4), results in a single equation for the effective radius retrieval from measurements of $R_{1}$ and $R_{2}$. This equation can be easily solved numerically (Press et al., 1992). The obtained value of $a_{\text {ef }}$ is used to calculate the optical thickness via Eq. (1), where the liquid water path is determined directly from Eq. (9) with account for Eq. (5). The values $r$ and $N$ are found using Eq. (1). Interestingly, it is possible to obtain the spherical albedo $r$ from bi-directional reflectance measurements.

\section{The cloud top height determination}

The determination of the cloud top height using SACURA is based on measurements of the top-ofatmosphere (TOA) reflection function $R$ in the oxygen A-band (Kokhanovsky et al., 2004, 2005a,b; Rozanov and Kokhanovsky, 2004; Rozanov et al., 2004). The cloud reflection function is extremely sensitive to the cloud top height in the center of the oxygen absorption A-band (Yamomoto and Wark, 1961). To find the value of $h$, we first assume that the TOA reflectance $R$ can be presented in the form of a Taylor expansion around the assumed value of the cloud top height equal to $h_{0}$ :

$R(h)=R\left(h_{0}\right)+\sum_{i=1}^{\infty} a_{i}\left(h-h_{0}\right)^{i}$,

where $a_{i}=R^{(i)}\left(h_{0}\right) / i$ ! Here, $R^{(i)}\left(h_{0}\right)$ is the $i$-derivative of $R$ at the point $h_{0}$. The next step is the linearization, which is the standard technique in the inversion procedures (Rozanov et al., 1998; Rozanov and Kokhanovsky, 2004; Rozanov et al., 2004; Rodgers, 2000). We found that the function $R(h)$ is close to a linear one in a broad interval of the argument change (Kokhanovsky and Rozanov, 2004). Therefore, we neglect nonlinear terms in Eq. (13). Then it follows:

$R=R\left(h_{0}\right)+R^{\prime}\left(h_{0}\right)\left(h-h_{0}\right)$,

where $R^{\prime}=\frac{\mathrm{d} R}{\mathrm{~d} h}$. We assume that $R$ is measured at several wavelengths in the oxygen A-band. Then instead of the scalar quantity $R$ we can introduce the vector $\vec{R}_{\text {mes }}$ with components $\left(R\left(\lambda_{1}\right), R\left(\lambda_{2}\right), \ldots, R\left(\lambda_{n}\right)\right)$. The same applies to other scalars in Eq. (14).

Therefore, Eq. (14) can be written in the following vector form:

$\vec{y}=x \vec{a}$

where $\vec{y}=\vec{R}_{\text {mes }}-\vec{R}\left(h_{0}\right), \vec{a}=\vec{R}^{\prime}\left(h_{0}\right)$, and $x=h-h_{0}$. Note that both measurement and model errors are contained in Eq. (15). The solution $\hat{x}$ of the inverse problem is obtained by the minimizing the following cost function (Rodgers, 2000):

$\Phi=\|\vec{y}-x \vec{a}\|^{2}$,

where \|\| means the norm in the Euclid space of the correspondent dimension.

The value of $\hat{x}$, where the function $\Phi$ has a minimum can be presented as (see Eq. (15)):

$\hat{x}=\frac{(\vec{y}, \vec{a})}{(\vec{a}, \vec{a})}$,

where (,) denotes a scalar product in the Euclid space.

Therefore, from known values of the measured spectral reflection function $R_{\text {mes }}$ (and also values of the calculated reflection function $R$ and its derivative $R^{\prime}$ at $h=h_{0}$ ) at several wavelengths, the value of the cloud top height can be found from Eq. (17) and the equality: $h=\hat{x}+h_{0}$. The value of $h_{0}$ can be taken equal to $1.0 \mathrm{~km}$. This is a typical value for low level clouds (Feigelson, 1981). The main assumption in our derivation is that the dependence of $R$ on $h$ can be presented by a linear function on the interval $x$ (Kokhanovsky and Rozanov, 2004).

As a matter of fact, the SACURA code finds both the cloud top height $h$ and the cloud geometrical thickness $l$ simultaneously. This requires the minimization of the following cost function (see Eq. (16)):

$\Phi=\|\vec{y}-\hat{A} \vec{X}\|^{2}$.

The elements of the matrix $\hat{A}$ are correspondent weighting functions (Rozanov et al., 1998, 2002; Rodgers, 2000). The solution of the inverse problem is given by the vector-parameter $\vec{X}$. This vector has five components, which give corrections to the initially assumed cloud top height and cloud geometrical thickness, the correction to the initially assumed half-width of the spectrometer spectral response function, the shift parameter, and the squeeze parameter. Further details are given by Rozanov and Kokhanovsky (2004). 
We have developed two versions of the retrieval algorithm. One is based on the exact radiative transfer calculations of the reflection function $R$ and its derivatives $R^{\prime}$ and another one is based on the approximate representation of $R$ by the following equation (Kokhanovsky and Rozanov, 2004):

$R=R_{0}+T_{1} R_{\mathrm{c}} T_{2}$,

where $R_{0}$ gives the reflection function of the atmospheric layer above the cloud and $R_{\mathrm{c}}$ is the cloud reflection. Functions $T_{i}(i=1,2)$ give transmission coefficients from the sun to a cloud and from the cloud to a satellite, respectively. Approximate analytical equations for all functions in Eq. (19) are given by Kokhanovsky and Rozanov (2004). We have used the correlated $k$-distribution method to account for the high-frequency oscillations of the oxygen molecule absorption cross-section $\sigma_{\mathrm{a}}$ (Lacis and Oinas, 1991; Buchwitz, 2000; Buchwitz et al., 2000). The temperature and pressure dependence of $\sigma_{\mathrm{a}}$ for a given location of measurements was accounted for using the standard atmosphere model built in SCIATRAN (Rozanov et al., 2002). We have used the most recent version of the HITRAN molecular spectroscopic database to get data on cross-sections $\sigma_{\mathrm{a}}$ (Rothman et al., 2003).

For the operational retrievals, we have used the retrieval technique based on the approximate representation of $R$ (see Eq. (17)). It allows to speed up the retrieval process considerably. We underline that the SACURA is capable to retrieve the cloud geometrical thickness as well. However, the meaning of this parameter for multi-layered cloud systems is not clear.

\section{The phase index}

The thermodynamic state of clouds is an important parameter for cloud physics, climate problems and aviation safety. It is known that spectral properties of ice differ significantly in the spectral range $1500-1700 \mathrm{~nm}$ from those of water (Knap et al., 2002; Acarreta et al., 2004). Therefore, we introduce the phase index (PI) $\alpha$ :

$\alpha=\frac{R(1550 \mathrm{~nm})}{R(1670 \mathrm{~nm})}$,

to characterize the thermodynamic state of a cloud. Low values of this parameter correspond to ice clouds.

We find using radiative transfer calculations that the value of $\alpha$ is in the range $0.7-1.0$ for water clouds and it is in the range $0.5-0.7$ for ice clouds. Calculations were performed using the modified asymptotic equations given by Kokhanovsky and Rozanov (2003) in the assumptions that both crystals and droplets have the spherical shape. It was assumed that the cloud optical thickness is varied in the range 5-30 for both water and ice clouds. The effective radius of droplets in water clouds and also ice crystals was changed in the range 5-30 microns. Studies performed by Kokhanovsky et al. (2005b) show that the increase of $a_{\text {ef }}$ leads to the increase of the separation of both cloud types. Note that ice crystals have highly irregular shape and also the size is often in the range 100-500 microns as compared to just 10 microns for warm water clouds. This will enhance differences even further. Therefore, the PI indeed can be used as an indicator of the cloud phase. More discussions on the phase index are given by Kokhanovsky et al. (2005b).

Obviously, we have for clear sky over black surfaces: $R(1550 \mathrm{~nm})>R(1670 \mathrm{~nm})$, and, therefore, $\alpha>1$. Some highly reflecting soils could have values of $\alpha=0.5-0.7$ similar to crystalline clouds. However, these pixels are screened out using information on the derived cloud top height, the cloud optical thickness or both.

\section{Results of retrievals}

The SACURA has been applied to all nadir data of the SCAnning Imaging Absorption spectrometer for Atmospheric ChartographY (SCIAMACHY) on board ENVISAT. SCIAMACHY is a moderate resolution imaging spectrometer. It composed of a mirror system, a telescope, a spectrometer, and thermal and electronic sub systems. Spectra are recorder simultaneously from 214 to $1750 \mathrm{~nm}$ and also in two smaller spectral regions 1940-2040 and 2265-2380 nm. Spectral resolution depends on the channel: $0.21 \mathrm{~nm}(214-314 \mathrm{~nm}), 0.22 \mathrm{~nm}$ $(309-4004 \mathrm{~nm}), \quad 0.47 \mathrm{~nm} \quad(392-605 \mathrm{~nm}), \quad 0.42 \mathrm{~nm}$ $(598-790 \mathrm{~nm}), 0.55 \mathrm{~nm}(776-1056 \mathrm{~nm}), 1.56 \mathrm{~nm}(991-$ $1750 \mathrm{~nm}), 0.21 \mathrm{~nm}(1940-2040 \mathrm{~nm}), 0.24 \mathrm{~nm} \quad(2260$ $2384 \mathrm{~nm}$ ). Measurements of SCIAMACHY are performed in nadir, limb, and solar/lunar occultation modes (14 orbits per day). The ground pixel size of SCIAMACHY varies depending on the spectral channel used. We used pixels with the size $30 * 60 \mathrm{~km}^{2}$ in the retrievals presented in this paper. Further details related to SCIAMACHY are given by Bovensmann et al. (1999). The results of retrievals and correspondent files are freely available at www.iup.physik.uni-bremen.de/scia-arc.

The results of cloud top height retrievals using the oxygen A-band depth technique are given in Fig. 1 for September 17th, 2003. All 14 SCIAMACHY orbits available for this date are shown. The enhanced picture of every state is given at our website. The instrument operates in the limb mode in the case of gaps shown in the figure. Then nadir data are not available. White colour corresponds to cloud top heights above $15 \mathrm{~km}$ and the situation with no colours in the SCIAMACHY ground scenes corresponds to a clear sky conditions. It follows from Fig. 1 that the number of clear sky situations is very limited in comparison with cloudy scenes. 


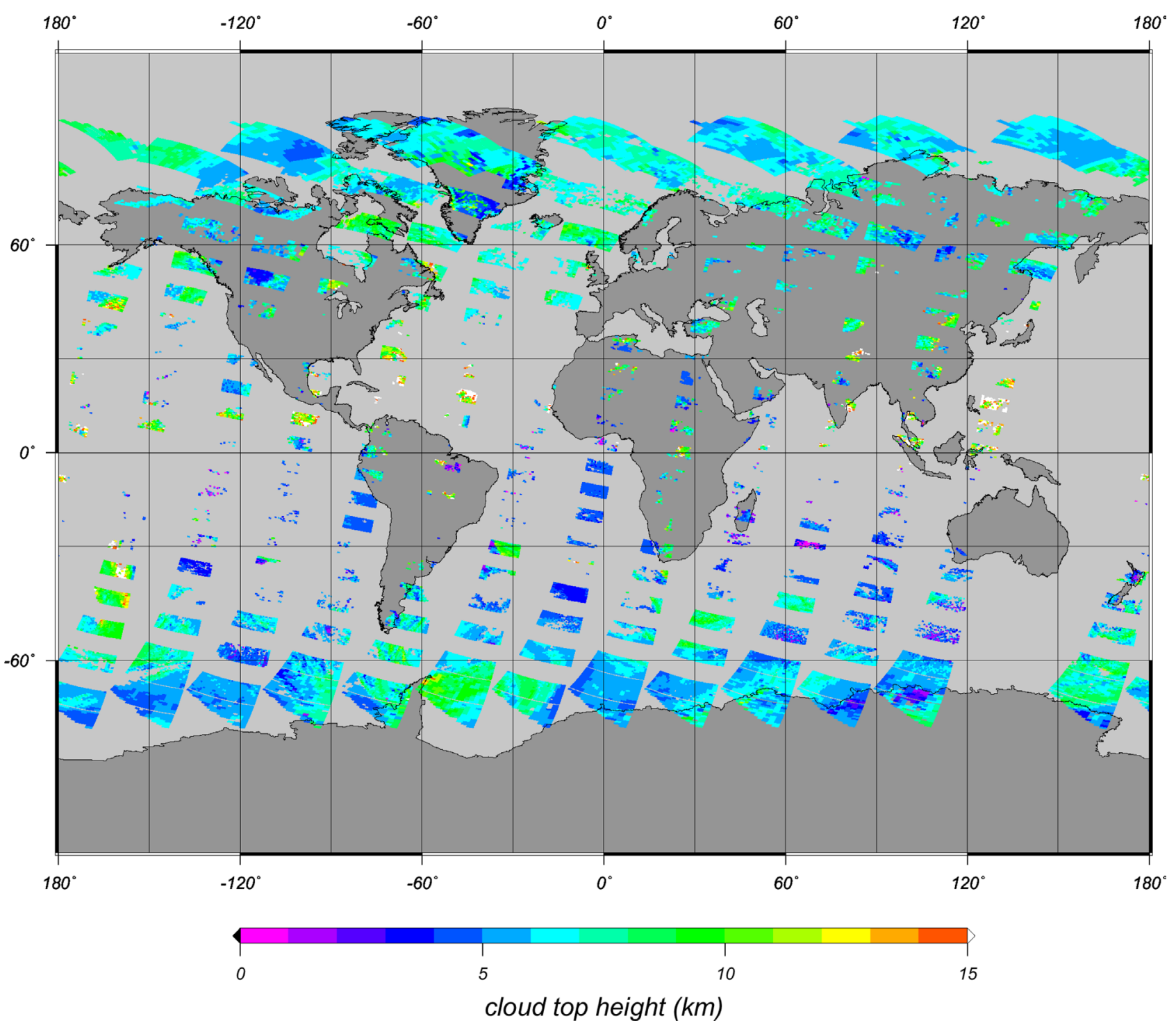

Fig. 1. The global cloud top height map for September 17th, 2003 as derived by SACURA using SCIAMACHY data.

This really should be the case because cloudiness covers approximately $60 \%$ of the terrestrial surface at any given time. So cloud retrieval algorithms are crucial for SCIAMACHY trace gases columns retrievals.

It follows from Fig. 1 that most of high-altitude clouds are located in the region $0-30^{\circ} \mathrm{N}$ degrees. This coincides with general knowledge on global cloud vertical distribution available at present. Also hurricane activity is most pronounced namely in this region. In particular SCIAMACHY captured the Hurricane Isabel in the scene shown in Fig. 1. The Isabel occupies a part of the scene located North-East of Florida $\left(30^{\circ} \mathrm{N}, 70^{\circ} \mathrm{E}\right)$ for the SCIAMACHY orbit 08095. A small portion of the cloud system associated with Isabel is also seen in the next state (in direction to the South). The distribution of the cloud top heights with the latitude for the orbit 08095 of ENVISAT, which contains data for Isabel, is given in Fig. 2. It follows that Isabel reaches height $18 \mathrm{~km}$. This is quite unusual for hurricanes at such latitudes to penetrate to such heights.

To confirm our finding we analysed SCIAMACHY limb state just before the hurricane. Then SCIAM$\mathrm{ACHY}$ has performed a vertical scan of the hurricane approaching it from the north. In particular, we plot the colour ratio $c=N(1050 \mathrm{~nm}) / N(750 \mathrm{~nm})$, where $N(\lambda)$ is the backscattered signal at the correspondent wavelength $\lambda$ normalized at its background value at $36 \mathrm{~km}$, in Fig. 3. It follows that this ratio is constant until the height $18 \mathrm{~km}$. Then it drops substantially. It can be explained by the fact that both ice crystals and water droplets scatter light almost independently at the wavelength in the spectral interval $400-1200 \mathrm{~nm}$. This is not the case for a clear atmosphere above the hurricane. Then the value of $c$ decreases (e.g., $c$ should be lower 


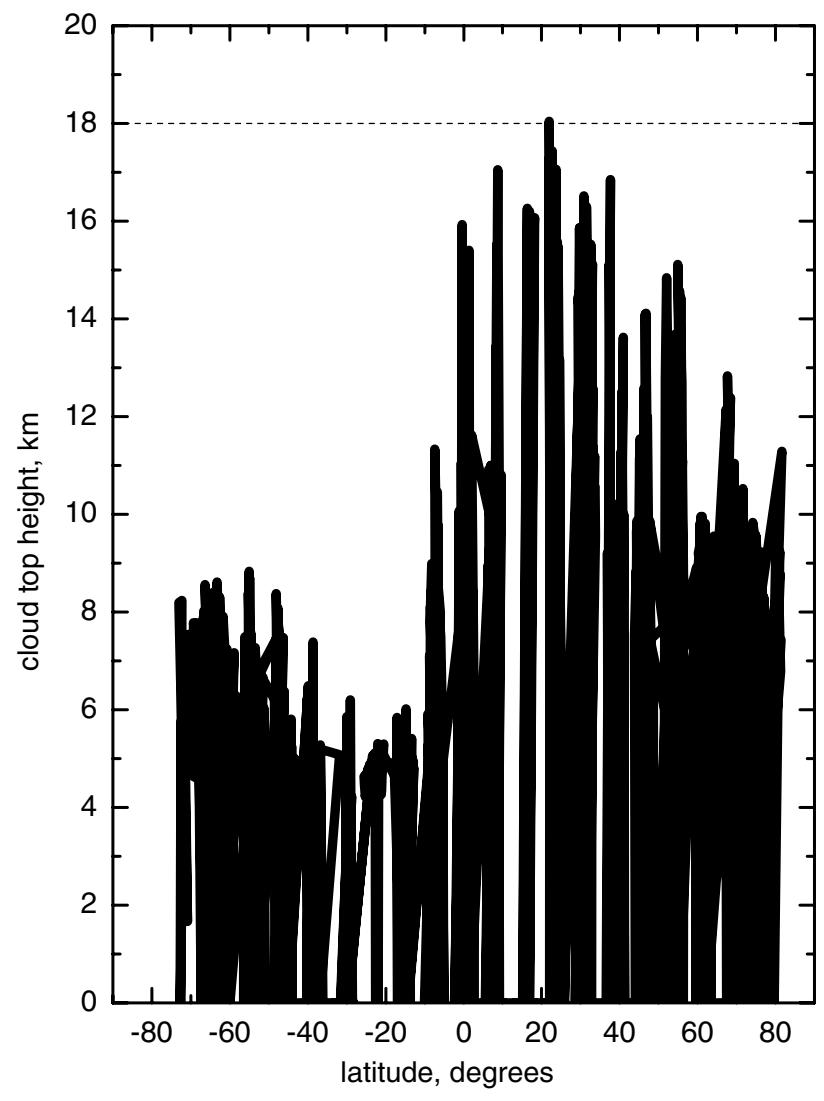

Fig. 2. The dependence of the cloud top height on the latitude for the orbit 08095 (September 17th, 2003) as derived by SACURA.

for both stratospheric aerosol and molecular scattering than for ice or water clouds). This is seen in experimental data presented in Fig. 3 as well. Our considerations confirm that the hurricane under study penetrates to a high altitude and signifies an important role of hurricanes in supplying water and also other lower atmosphere constitutes into stratosphere. Hurricanes could also alter the stratospheric dynamics (at least in a hurricane season).

Note that top of a hurricane should consist of ice crystals. This is confirmed by the phase index values obtained in this case. They are generally below 0.7. The phase index statistical distribution for a whole orbit 08095 is shown in Fig. 4(a). It follows that the phase index is in the range $0.35-1.0$. It has a bi-modal distribution. The mode positioned at values of PI $\alpha \approx 0.85$ is due to water clouds, and yet another mode located at the phase index close to 0.7 is due to crystalline clouds. The visual inspection of Fig. 4(a) confirms that the probability of ice cloud occurrence is at least 2 times smaller than that of water clouds for the orbit analysed, which is a reasonable estimate. The statistical distributions of cloud top heights for the same orbit is shown in Fig. 4(b). It follows that this distribution is bi-modal with most frequent values of the cloud top height located at 5 and $7 \mathrm{~km}$. The statistical distribution of the derived

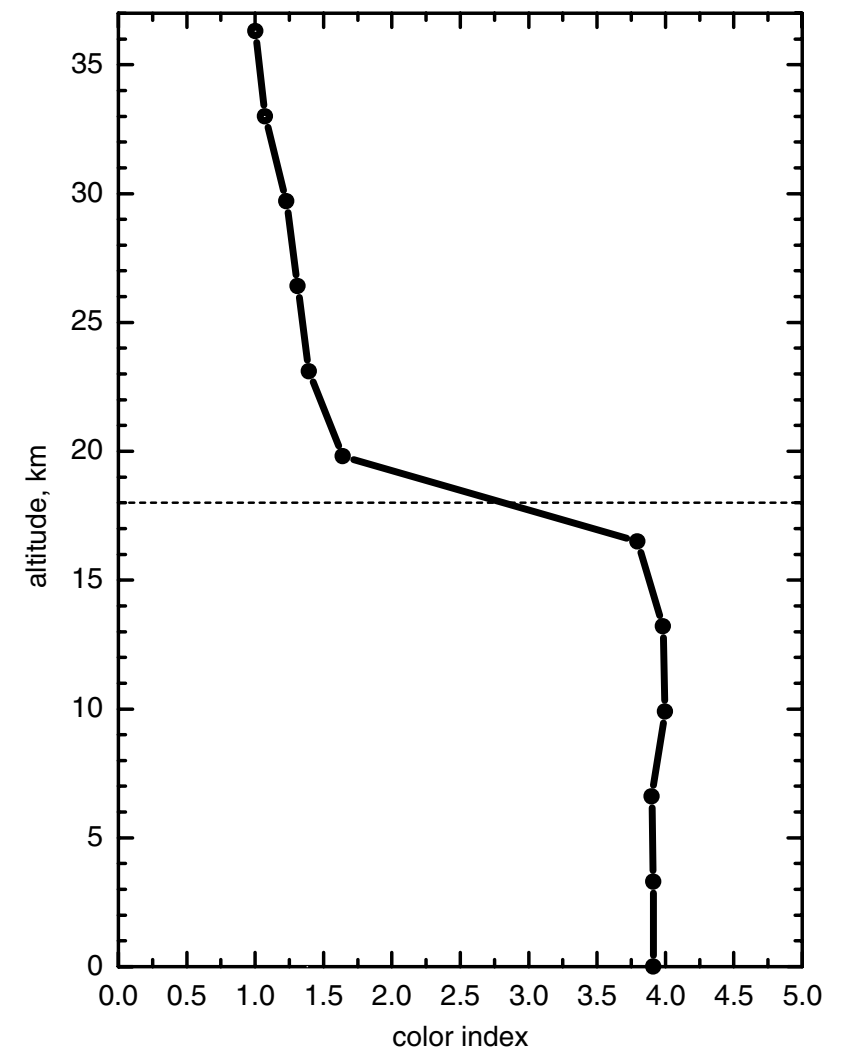

Fig. 3. The limb profile of the hurricane Isabel area as measured by SCIAMACHY (the vertical resolution $\sim 3 \mathrm{~km}$ ).

optical thickness is shown in Fig. 4(c). The most frequent values of $\tau$ are around 5. The dependence as shown in Fig. 4(c) was also found analysing a great number of ground-based measurements by Trishchenko et al. (2001). This indirectly confirms the results obtained. SACURA does not retrieve values of $\tau \geqslant 100$ because of insensitivity of the cloud reflection function to cloud optical thickness for extremely thick cloud systems. So retrieved values of $\tau$ larger than 100 are artificially set to $\tau=100$. It follows from Fig. 4(c) that it is about 50 such cases in the orbit analysed. Most of them are related to the hurricane Isabel. The liquid water path distribution obtained for the same orbit is given in Fig. 4(d). Large values of LWP are again associated with the hurricane. However, we see that most frequent values of LWP are located in the region $50-70 \mathrm{~g} / \mathrm{m}^{2}$. The average value of LWP is around $100 \mathrm{~g} / \mathrm{m}^{2}$. This closely coincides with average annual values of LWP derived by Han et al. (1994) $\left(87 \mathrm{~g} / \mathrm{m}^{2}\right.$ over ocean) from AVHRR data on board NOAA-9 and NOAA-10.

The measured top-of-atmosphere reflection function statistical distribution at the wavelength $440 \mathrm{~nm}$ is given in Fig. 5. It is obtained by the normalization of the nadir backscattered intensity by the solar light flux reflected by the white diffuse plate present in SCIAMACHY. The values of $R$ in the range $0.3-1.0$ are associated with 

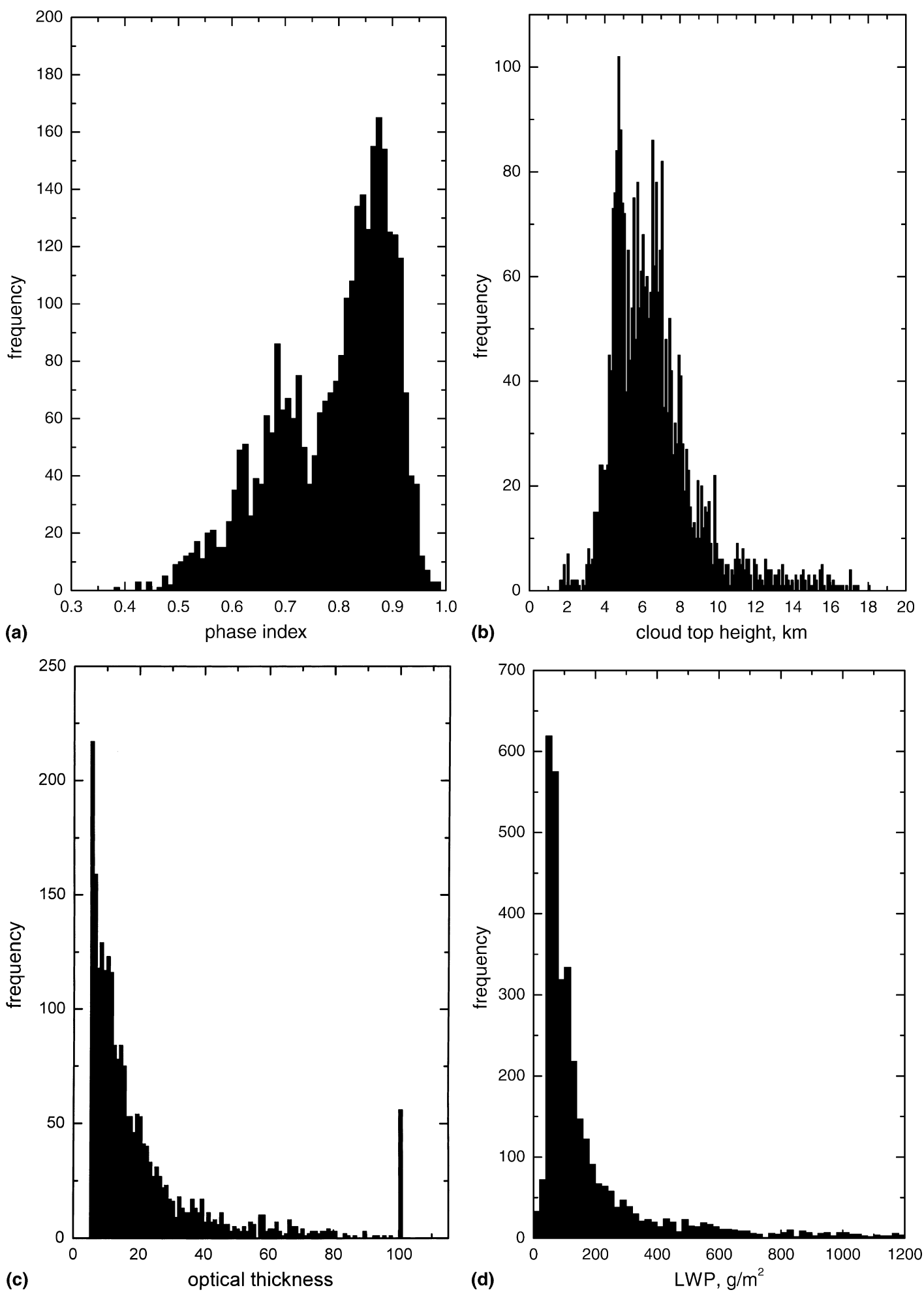

Fig. 4. The phase index (a), the cloud top height (b), the optical thickness (c), the liquid water path (d) statistical distributions for the orbit 08095 (September 17th, 2003) as derived from SACURA using SCIAMACHY data.

highly reflecting surfaces. So the study of the spatial distribution of the reflection function is a convenient tool to access the reflective properties of observed surfaces and atmospheric layers from space.
It follows that values of $R$ below 0.1 never occur. This is because light reflectance from ocean/land, Rayleigh scattering, and background aerosol are always present in the satellite-detected signal at wavelengths used. 


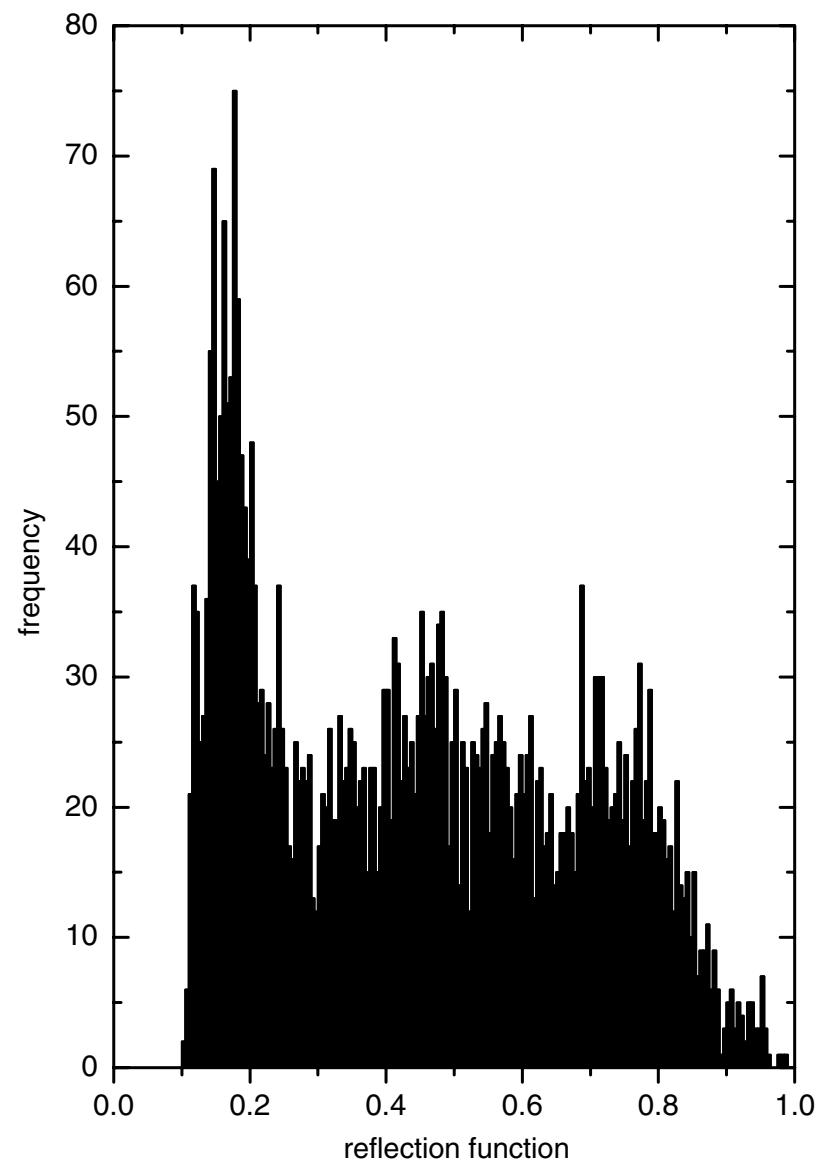

Fig. 5. The statistical distribution of the reflection function for the orbit 08095 (September 17th, 2003) as measured by SCIAMACHY (the spatial resolution $\sim 30 * 60 \mathrm{~km}^{2}$ ).

Three modes are clearly seen in Fig. 5. They can be associated with cloudless conditions (the mode for smallest values of $R$ ) and two modes for thin and thick (most probably, multilayered) clouds. It was found that the average value of $R$ for the orbit studied is 0.45 with the coefficient of variance $50 \%$.

\section{Conclusions}

The system of analytical equations has been developed, which was used to retrieve the effective radius of droplets and the liquid water path of optically thick clouds $(\tau \geqslant 5)$. Also other cloud characteristics are found. They are the optical thickness, the cloud albedo, the droplet column concentration, the phase index, the cloud top height and the cloud geometrical thickness.

We give maps of the optical thickness, the effective radius, the liquid water path, the phase index, $R(443 \mathrm{~nm})$, and cloud mask at our website for a particular orbit. These files are accompanied by the numerical data.

The cloud top height is determined using spectral measurements in the 755-770 nm oxygen A-band and the phase index is determined using differences in the refractive index of liquid water as compared to ice in the spectral range $1550-1670 \mathrm{~nm}$. Such spectral measurements are routinely performed by the SCIAMACHY instrument (Bovensmann et al., 1999).

One important feature of the algorithm proposed is its simplicity and a high speed of retrieval procedures. It can be used as an integrated and autonomous part of operational cloud satellite retrieval schemes. It is characterized by a high accuracy and have important advantages as compared to the standard fitting methods used in up-to-date cloud retrieval schemes (especially if the speed of calculations is a concern as it is often the case in the analysis of large databases). The accuracy of the retrievals was checked by Kokhanovsky et al. (2004), Rozanov and Kokhanovsky (2004), and Kokhanovsky et al. (2005a,b). Nauss and Kokhanovsky (2005) found that SACURA produces results comparable to other algorithms (e.g., JAXA and NASA algorithms as applied to MODerate resolutIon Spectrometer (MODIS) data).

Although both aerosol and molecular scattering and absorption are neglected, their influence is minimized by a careful selection of spectral channels. Also, the influence of molecular and aerosol scattering is of importance mostly for $\tau<5$ (Wang and King, 1997). This range of optical thicknesses is out of scope of the applicability of the algorithm described. The sensitivity of the top-of-atmosphere radiance to the ground albedo is low for thick clouds. So we assume that the albedo of ground is equal to zero.

The applicability of the algorithm proposed for the optical thickness retrieval is limited both from the side of small $(\tau<5)$ and large $(\tau \geqslant 100)$ values of the optical thickness (Kokhanovsky et al., 2003). Note that the dependence of $R$ on $\tau$ disappears as $\tau \rightarrow \infty$. However, the appearance of such clouds in the Earth's atmosphere is rare as indicated by Trishchenko et al. (2001). For the effective radius retrieval, however, there is no upper boundary for large $\tau$. This is due to the fact that the reflection of light depends on the size of droplets even for an infinitely thick absorbing cloud. The footprint of the SCIAMACHY instrument is $30 * 60 \mathrm{~km}^{2}$ for all studies performed here. This underlines the fact that thin clouds retrievals with this instrument is difficult to handle due to possible broken cloud conditions associated with small values of $\tau$.

In conclusion, note that the colour index $c$ can be used for mapping convective cloud events using SCIAMACHY limb measurements (see Fig. 3). This is of importance for a number of problems and especially for troposphere-stratosphere exchange studies.

SACURA code is available online as a part of the radiative transfer solver SCIATRAN (Rozanov et al., 2005) (see www.iup.physik.uni-bremen.de/sciatran). 


\section{Acknowledgements}

This work was supported by the DFG Project BU 688/8-1. The authors are grateful to H. Bovensman, K. Bramstedt, M. Buchwitz, and H. Schroeter for their help and important advices. Some data shown here were calculated on HLRN (High-Performance Computer Center North, Germany). Services and support are gratefully acknowledged.

\section{References}

Acarreta, J.R. et al. First retrieval of cloud phase from SCIAMACHY spectra around $1.6 \mu \mathrm{m}$. Atmos. Res. 72, 89-105, 2004.

Arking, A., Childs, J.D. Retrieval of clouds cover parameters from multispectral satellite images. J. Appl. Meteorol. 24, 323-333, 1985.

Bovensmann, H. et al. SCIAMACHY: mission objectives and measurement modes. J. Atmos. Sci. 56, 127-150, 1999.

Bovensmann, H. et al. SCIAMACHY on ENVISAT: in flight optical performance and first results. Proc. SPIE 5235, 160-173, 2004.

Buchwitz, M. Strahlungstransport- und Inversions- Algorithmen zur Ableitung Atmospharischer Spurengasinformationen aus Erdfernerkundungsmessungen in Nadirgeometrie im ultravioletten bis nahinfraroten Spektralbereich am Beispiel SCIAMACHY. PhD thesis, Bremen, Bremen University, 2000.

Buchwitz, M., Rozanov, V.V., Burrows, J.P. A correlated-k distribution scheme for overlapping gases suitable for retrieval of atmospheric constituents from moderate resolution radiance measurements in the visible/near-infrared spectral region. J. Geophys. Res. D105, 15247-15262, 2000.

Deschamps, P.-Y. et al. The POLDER Mission: Instrument characteristics and scientific objectives. IEEE Trans. Geosci. Remote Sens. 32, 598-614, 1994.

Feigelson, E.M. (Ed.). Radiation in a Cloudy Atmosphere. Gidrometeoizdat, Leningrad, p. 280, 1981.

Fischer, J., et al. Cloud top pressure, MERIS Algorithm Theoretical Basis Document No ATBD 2.3. Berlin, Free University of Berlin, 2000.

Goloub, P. et al. Cloud thermodynamical phase classification from the POLDER spaceborne instrument. J. Geophys. Res. D105, 14747$14759,2000$.

Germogenova, T.A. Some formulas to solve the transfer equation in the plane layer problem, in: Stepanov, B.I. (Ed.), Spectroscopy of Scattering Media. AN BSSR, Minsk 1963, pp. 36-41 1961.

Germogenova, T.A. Some formulas to solve the transfer equation in the plane layer problem. In: Stepanov, B.I. (Ed.), Spectroscopy of Scattering Media. AN BSSR, Minsk, pp. 36-41, 1963.

Han, Q. et al. Near global survey of effective droplet radii in liquid water clouds using ISCCP data. J. Climate 7, 465-497, 1994.

King, M.D. Determination of the scaled optical thickness of clouds from reflected soar radiation measurements. J. Atmos. Sci. 44, 1734-1751, 1987.

King, M.D. et al. Remote sensing of cloud, aerosol, and water vapour properties from the moderate resolution imaging spectrometer (MODIS). IEEE Trans. Geosci. Remote Sens. 30, 2-27, 1992.

Knap, W.H., Stammes, P., Koelemeijer, R.B.A. Cloud thermodynamic phase determination from near-infrared spectra of reflected sunlight. J. Atmos. Sci. 59, 83-96, 2002.

Koelemeijer, R.B.A., Stammes, P., Hovenier, J.W., De Haan, J.F. A fast method for retrieval of cloud parameters using oxygen A band measurements from GOME. J. Geophys. Res. 106, 3475-3490, 2001.
Koelemeijer, R.B.A., Stammes, P., Hovenier, J.W., De Haan, J.F. Global distributions of effective cloud fraction and cloud top pressure derived from oxygen A band spectra measured by the Global Ozone Monitoring Experiment: comparison to ISCCP data. J. Geophys. Res., 107, 2002.

Kokhanovsky, A.A. et al. A semianalytical cloud retrieval algorithm using backscattered radiation in $0.4-2.4 \mu \mathrm{m}$ spectral region. J. Geophys. Res. D108, 4008, 2003.

Kokhanovsky, A.A., Rozanov, V.V. The reflection function of optically thick weakly absorbing layers: a simple approximation. J. Quant. Spectrosc. Rad. Transfer 77, 165-175, 2003.

Kokhanovsky, A.A. Light Scattering Media Optics. Springer-Praxis, Berlin, 2004a.

Kokhanovsky, A.A. Optical properties of terrestrial clouds. Earth-Sci. Rev. 64, 189-241, 2004b.

Kokhanovsky, A.A. Reflection of light from nonabsorbing semiinfinite cloudy media: a simple approximation. J. Quant. Spectrosc. Rad. Transfer 85, 25-33, 2004c.

Kokhanovsky, A.A., Rozanov, V.V. The physical parameterization of the top-of-atmosphere reflection function for a cloudy atmosphere - underlying surface system: the oxygen A-band case study. J. Quant. Spectrosc. Rad. Transfer 85, 35-55, 2004.

Kokhanovsky, A.A. et al. The determination of a cloud altitude using SCIAMACHY on board ENVISAT. IEEE Trans. Geosci. Remote Sens. Lett. 1, 211-214, 2004.

Kokhanovsky, A.A., et al. The semianalytical cloud retrieval algorithm for SCIAMACHY. II. The application to MERIS and SCIAMACHY data. Atmos. Chem., Phys. Discuss. (submitted) 2005a.

Kokhanovsky, A.A., et al. The semianalytical cloud retrieval algorithm for SCIAMACHY. I. The validation. Atmos. Chem., Phys. Discuss. (in press) 2005b.

Kondratyev, K.Y., Binenko, V.I. Impact of Cloudiness on Radiation and Climate. Gidrometeoizdat, Leningrad, 1984.

Kuji, M., Nakajima, T. Retrieval of cloud geometrical parameters using remote sensing data, in: Utah: CD-Proceedings of 11th Conference on Atmospheric Radiation, JP1.7 2002.

Kuze, A., Chance, K.V. Analysis of cloud top height and cloud coverage from satellites using the $\mathrm{O}_{2} \mathrm{~A}$ and B bands. J. Geophys. Res. 99, 14481-14491, 1994.

Lacis, A.A., Oinas, V. A description of the correlated k distribution method for modeling nongray gaseous absorption, thermal emission, and multiple scattering in vertically inhomogeneous atmospheres. J. Geophys. Res. D96, 9027-9063, 1991.

Liou, K.N. Radiation and Cloud Processes in the Atmosphere. Oxford University Press, Oxford, 1992.

Loeb, N.G., Davies, R. Observational evidence of plane parallel model biases: Apparent dependence of cloud optical depth on solar zenith angle. J. Geophys. Res. 101, 1621-1634, 1996.

Loeb, N.G., Coakley Jr., J.A. Inference of marine stratus cloud optical depths from satellite measurements: does 1D theory apply?. J. Atmos. Sci. 11, 215-233, 1998.

Nakajima, T., King, M.D. Determination of the optical thickness and effective particle radius of clouds from reflected solar radiation measurements Part I. Theory. J. Atmos. Sci. 47, 18781893, 1990.

Nakajima, T. et al. Determination of the optical thickness and effective particle radius of clouds from reflected solar radiation measurements. Part II. Marine stratocumulus observations. J. Atmos. Sci. 48, 728-750, 1991.

Nakajima, T.Y. et al. Optimization of the Advanced Earth Observing Satellite II Global Imager channels by use of the raduiative transfer calculations. Appl. Opt. 37, 3149-3163, 1998.

Nakajima, T.Y. et al. Cloud properties observed by Global Imager (GLI). Proc. SPIE 5570, 22-32, 2005.

Nauss, T., Kokhanovsky, A.A. The intercomparison of selected cloud retrieval algorithms. Atmos. Res. (in press) 2005. 
Pilewskie, P., Twomey, S. Cloud phase discrimination by reflectance measurements near 1.6 and $2.2 \mu \mathrm{m}$. J. Atmos. Sci. 44, 3419-3421, 1987a.

Pilewskie, P., Twomey, S. Discrimination of ice from water in clouds by optical remote sensing. Atmos. Res. 21, 113-122, $1987 \mathrm{~b}$.

Platnick, S. Approximations for horizontal photon transport in cloud remote sensing problems. J. Quant. Spectr. Rad. Transfer 68, 7599, 2001.

Platnick, S. et al. A solar reflectance method for retrieving the optical thickness and droplet size of liquid water clouds over snow and ice surfaces. J. Geophys. Res. D14 (106), 15185-15199, 2001.

Platnick, S. et al. The MODIS cloud products: algorithms and examples from Terra. IEEE Trans. Geosci. Remote Sens. 41, 459-473, 2003.

Press, W.H. et al. Numerical Receipes in FORTRAN. The Art of Scientific Computing, second ed. Cambridge University Press, Cambridge, 1992.

Rodgers, C. Inverse Methods for Atmospheric Sounding: Theory and Practice. World Scientific, Singapore, 2000.

Rossow, W.B. Measuring cloud properties from space: a review. J. Climate 2, 419-458, 1989.

Rossow, W.B., Schiffer, R.A. Advances in understanding clouds from ISCCP. Bull. Am. Meteor. Soc. 80, 2261-2287, 1999.

Rothman, L.S. et al. The HITRAN molecular spectroscopic database: edition of 2000 including updates through 2001. J. Quant. Spectrosc. Radiat. Transfer 82, 5-44, 2003.

Rozanov, A.V., et al. SCIATRAN 2.0- a new radiative transfer model for geophysical applications in the $175-2400 \mathrm{~nm}$ spectral region. Adv. Space Res. (this issue) 2005.

Rozanov, V.V., Kurosu, T., Burrows, J.P. Retrieval of atmospheric constituents in the UV-Visible: a new quasi-analytical approach for the calculation of weighting functions. J. Quant. Spectrosc. Radiat. Transfer 60, 277-299, 1998.
Rozanov, V.V., Buchwitz, M., Eichmann, K.-U., de Beek, R., Burrows, J.P. SCIATRAN - a new radiative transfer model for geophysical applications in the $240-2400 \mathrm{~nm}$ spectral range: The pseudo-spherical version. Adv. Space Res. 29, 1831-1835, 2002.

Rozanov, V.V., Kokhanovsky, A.A. Semi-analytical cloud retrieval algorithm as applied to the cloud top altitude and the cloud geometrical thickness determination from top of atmosphere reflectance measurements in the oxygen absorption bands. J. Geophys. Res. 109, D05202, 2004.

Rozanov, V.V., Kokhanovsky, A.A., Burrows, J.P. The determination of cloud altitudes using GOME reflectance spectra: multilayered cloud systems. IEEE Trans. Geosci. Remote Sens. 42, 1009-1017, 2004.

Rozenberg, G.V. et al. The determination of optical characteristics of clouds from measurements of the reflected solar radiation using data from the Sputnik "KOSMOS-320". Izvestiya Acad. Sci. USSR, Fizika Atmos. Okeana 10, 14-24, 1978.

Slingo, A. A GCM parameterization for the shortwave radiative properties of water clouds. J. Atmos. Sci. 46, 1419-1427, 1989.

Trishchenko, A.P. et al. Cloud optical depth and TOA fluxes: comparison between satellite and surface retrievals from multiple platforms. Gephys. Res. Lett. 28, 979-982, 2001.

Van de Hulst, H.C. Multiple Light Scattering: Tables, Formulas and Applications. Academic Press, 1980.

Wang, M., King, M.D. Correction of Rayleigh scattering effects in cloud optical thickness retrievals. J. Geophys. Res. D22 (102), 25915-25926, 1997.

Yamomoto, G., Wark, D.Q. Discussion of letter by A. Hanel: determination of cloud altitude from a satellite. J. Geophys. Res. 66, 3596, 1961. 